\title{
THE SEMI-INFINITE $q$-BOSON SYSTEM WITH BOUNDARY INTERACTION
}

\author{
J.F. VAN DIEJEN AND E. EMSIZ
}

\begin{abstract}
Upon introducing a one-parameter quadratic deformation of the $q$-boson algebra and a diagonal perturbation at the end point, we arrive at a semi-infinite $q$-boson system with a two-parameter boundary interaction. The eigenfunctions are shown to be given by Macdonald's hyperoctahedral Hall-Littlewood functions of type $B C$. It follows that the $n$-particle spectrum is bounded and absolutely continuous and that the corresponding scattering matrix factorizes as a product of two-particle bulk and one-particle boundary scattering matrices.
\end{abstract}

\section{INTRODUCTION}

The $q$-boson system [BIK] is a lattice discretization of the one-dimensional quantum nonlinear Schrödinger equation [G2, G, KBI, Mt, [S built of particle creation and annihilation operators representing the $q$-oscillator algebra [KS, Ch. 5]. Its $n$-particle eigenfunctions are given by Hall-Littlewood functions $\mathrm{T}, \mathrm{K}, \mathrm{DE}$. In the present paper we study a system of $q$-bosons on the semi-infinite lattice with boundary interactions, in the spirit of previous works concerned with the quantum nonlinear Schrödinger equation on the half-line G1, GLM, [HL, CC, TW].

Specifically, by introducing at the end point creation and annihilation operators representing a quadratic deformation of the $q$-oscillator algebra together with a diagonal perturbation, we arrive at the hamiltonian of a $q$-boson system on the semi-infinite integer lattice endowed with a two-parameter boundary interaction. By means of an explicit formula for the action of the hamiltonian in the $n$-particle subspace, it is deduced that the Bethe Ansatz eigenfunctions are given by Macdonald's three-parameter Hall-Littlewood functions with hyperoctahedral symmetry associated with the $B C$-type root system [M], $\S 10]$.

It follows that the $q$-boson system fits within a large class of discrete quantum models with bounded absolutely continous spectrum for which the scattering behaviour was determined in great detail by means of stationary phase techniques $[\mathrm{R}, \mathrm{D} 3$. In particular, the $n$-particle scattering matrix is seen to factorize as a product of explicitly computed two-particle bulk and one-particle boundary scattering matrices.

Date: May 2013.

Work was supported in part by the Fondo Nacional de Desarrollo Científico y Tecnológico (FONDECYT) Grants \# 1130226 and \# 11100315, and by the Anillo ACT56 'Reticulados y Simetrías' financed by the Comisión Nacional de Investigación Científica y Tecnológica (CONICYT). 


\section{SEMI-INFINITE $q$-BOSON SYSTEM}

Let

$$
\mathcal{F}:=\bigoplus_{n \in \mathbb{N}} \mathcal{F}\left(\Lambda_{n}\right)
$$

denote the algebraic Fock space consisting of finite linear combinations of $f_{n} \in$ $\mathcal{F}\left(\Lambda_{n}\right), n \in \mathbb{N}:=\{0,1,2, \ldots\}$, where $\mathcal{F}\left(\Lambda_{n}\right)$ stands for the space of functions $f: \Lambda_{n} \rightarrow \mathbb{C}$ on the set of partitions of length at most $n$ :

$$
\Lambda_{n}:=\left\{\lambda=\left(\lambda_{1}, \ldots, \lambda_{n}\right) \in \mathbb{N}^{n} \mid \lambda_{1} \geq \lambda_{2} \geq \cdots \geq \lambda_{n}\right\}
$$

with the additional convention that $\Lambda_{0}:=\{0\}$ and $\mathcal{F}\left(\Lambda_{0}\right):=\mathbb{C}$. For $l \in \mathbb{N}$, we introduce the following actions on $f \in \mathcal{F}\left(\Lambda_{n}\right) \subset \mathcal{F}$ :

$$
\left(\beta_{l} f\right)(\lambda):=f\left(\beta_{l}^{*} \lambda\right) \quad\left(\lambda \in \Lambda_{n-1}\right)
$$

if $n>0$ and $\beta_{l} f:=0$ if $n=0$,

$$
\begin{aligned}
\left(\beta_{l}^{*} f\right)(\lambda) & :=\left\{\begin{array}{ll}
{\left[m_{l}(\lambda)\right]\left(1-c \delta_{l} q^{m_{0}(\lambda)-1}\right) f\left(\beta_{l} \lambda\right)} & \text { if } m_{l}(\lambda)>0 \\
0 & \text { otherwise }
\end{array} \quad\left(\lambda \in \Lambda_{n+1}\right),\right. \\
\left(q^{N_{l}+k} f\right)(\lambda) & :=q^{m_{l}(\lambda)+k} f(\lambda) \quad\left(\lambda \in \Lambda_{n}\right),
\end{aligned}
$$

with $q, c \in \mathbb{R}$ such that $|q| \neq 0,1$ and $k \in \mathbb{Z}$. Here

$$
\delta_{l}:=\left\{\begin{array}{ll}
1 & \text { for } l=0, \\
0 & \text { otherwise }
\end{array}, \quad[m]:=\frac{1-q^{m}}{1-q}=\left\{\begin{array}{ll}
0 & \text { for } m=0 \\
1+q+\cdots+q^{m-1} & \text { for } m>0
\end{array},\right.\right.
$$

and the multiplicity $m_{l}(\lambda)$ counts the number of parts $\lambda_{j}, 1 \leq j \leq n$ of size $\lambda_{j}=l$ (so $m_{0}(\lambda), \lambda \in \Lambda_{n}$ is equal to $n$ minus the number of nonzero parts), while $\beta_{l}^{*} \lambda \in \Lambda_{n+1}$ and $\beta_{l} \lambda \in \Lambda_{n-1}$ stand for the partitions obtained from $\lambda \in \Lambda_{n}$ by inserting/deleting a part of size $l$, respectively (where it is assumed in the latter situation that $\left.m_{l}(\lambda)>0\right)$. It is clear from these definitions that $\beta_{l}, \beta_{l}^{*}$ and $q^{N_{l}+k}$ map $\mathcal{F}\left(\Lambda_{n}\right)$ into $\mathcal{F}\left(\Lambda_{n-1}\right), \mathcal{F}\left(\Lambda_{n+1}\right)$ and $\mathcal{F}\left(\Lambda_{n}\right)$, respectively (with the convention that $\mathcal{F}\left(\Lambda_{-1}\right)$ is the null space).

The operators in question represent a quadratic deformation of the $q$-boson field algebra at the boundary site $l=0$ parametrized by the constant $c$ :

$$
\begin{gathered}
\beta_{l} q^{N_{l}}=q^{N_{l}+1} \beta_{l}, \quad \beta_{l}^{*} q^{N_{l}}=q^{N_{l}-1} \beta_{l}^{*}, \\
\beta_{l} \beta_{l}^{*}=\left[N_{l}+1\right]\left(1-c \delta_{l} q^{N_{0}}\right), \quad\left[\beta_{l}, \beta_{l}^{*}\right]_{q}=1-c \delta_{l} q^{2 N_{0}}
\end{gathered}
$$

and preserving the ultralocality:

$$
\left[\beta_{l}, \beta_{k}\right]=\left[\beta_{l}^{*}, \beta_{k}^{*}\right]=\left[N_{l}, N_{k}\right]=\left[N_{l}, \beta_{k}\right]=\left[N_{l}, \beta_{k}^{*}\right]=\left[\beta_{l}, \beta_{k}^{*}\right]=0
$$

for $l \neq k$ (where $[A, B]:=A B-B A,[A, B]_{q}:=A B-q B A$, and $\left[N_{l}+r\right]:=$ $\left.\left(1-q^{N_{l}+r}\right) /(1-q)\right)$.

When interpreting the characteristic function $|\lambda\rangle \in \mathcal{F}\left(\Lambda_{n}\right)$ supported on $\lambda \in \Lambda_{n}$ as a state representing a configuration of $n$ particles on $\mathbb{N}$ such that $m_{l}(\lambda)$ particles are siting on the site $l \in \mathbb{N}$, it is clear that the operators $\beta_{l}$ and $\beta_{l}^{*}$ act as particle annihilation and creation operators:

$$
\beta_{l}|\lambda\rangle=\left\{\begin{array}{ll}
\left|\beta_{l} \lambda\right\rangle & \text { if } m_{l}(\lambda)>0 \\
0 & \text { otherwise }
\end{array}, \quad \beta_{l}^{*}|\lambda\rangle=\left[m_{l}(\lambda)+1\right]\left(1-c \delta_{l} q^{m_{0}(\lambda)}\right)\left|\beta_{l}^{*} \lambda\right\rangle,\right.
$$


while $q^{N_{l}}$ counts the number of particles at the site $l$ (as a power of $q$ ):

$$
q^{N_{l}}|\lambda\rangle=q^{m_{l}(\lambda)}|\lambda\rangle
$$

The dynamics of our $q$-boson system is governed by a hamiltonian built of left and right hopping operators together with a diagonal boundary term:

$$
\mathrm{H}_{q}=a\left[N_{0}\right]+\sum_{l \in \mathbb{N}}\left(\beta_{l+1} \beta_{l}^{*}+\beta_{l+1}^{*} \beta_{l}\right)
$$

$a \in \mathbb{R}$. This hamiltonian constitutes a well-defined operator on $\mathcal{F}$ (2.1) as for any $f \in \mathcal{F}\left(\Lambda_{n}\right)$ and $\lambda \in \Lambda_{n}$ the infinite sum $\left(\mathrm{H}_{q} f\right)(\lambda)$ contains only a finite number of nonvanishing terms.

\section{The $n$-PARTICLE HAMILTONIAN AND ITS EIGENFUNCTIONS}

By construction $\mathrm{H}_{q}(2.4)$ preserves the $n$-particle subspace $\mathcal{F}\left(\Lambda_{n}\right)$. The following proposition describes the action of the hamiltonian in this subspace explicitly.

Proposition 3.1 (n-Particle hamiltonian). For any $f \in \mathcal{F}\left(\Lambda_{n}\right)$ and $\lambda \in \Lambda_{n}$, one has that

$$
\begin{aligned}
& \left(H_{q} f\right)(\lambda)=a\left[m_{0}(\lambda)\right] f(\lambda)+ \\
& \sum_{\substack{1 \leq j \leq n \\
\lambda+e_{j} \in \Lambda_{n}}}\left(1-c \delta_{\lambda_{j}} q^{m_{0}(\lambda)-1}\right)\left[m_{\lambda_{j}}(\lambda)\right] f\left(\lambda+e_{j}\right)+\sum_{\substack{1 \leq j \leq n \\
\lambda-e_{j} \in \Lambda_{n}}}\left[m_{\lambda_{j}}(\lambda)\right] f\left(\lambda-e_{j}\right),
\end{aligned}
$$

where $e_{1}, \ldots, e_{n}$ refer to the unit vectors comprising the standard basis of $\mathbb{Z}^{n}$.

Proof. It is clear from the definitions that $\left(\left[N_{0}\right] f\right)(\lambda)=\left[m_{0}(\lambda)\right] f(\lambda)$, and that for any $l \in \mathbb{N}$ :

$$
\left(\beta_{l+1} \beta_{l}^{*} f\right)(\lambda)= \begin{cases}{\left[m_{l}(\lambda)\right]\left(1-c \delta_{l} q^{m_{0}(\lambda)-1}\right) f\left(\beta_{l+1}^{*} \beta_{l} \lambda\right)} & \text { if } m_{l}(\lambda)>0 \\ 0 & \text { otherwise }\end{cases}
$$

where $\beta_{l+1}^{*} \beta_{l} \lambda=\lambda+e_{j}$ with $j=\min \left\{k \mid \lambda_{k}=l\right\}$ (so $l=\lambda_{j}$ ), and

$$
\left(\beta_{l+1}^{*} \beta_{l} f\right)(\lambda)= \begin{cases}{\left[m_{l+1}(\lambda)\right] f\left(\beta_{l+1} \beta_{l}^{*} \lambda\right)} & \text { if } m_{l+1}(\lambda)>0, \\ 0 & \text { otherwise, }\end{cases}
$$

where $\beta_{l+1} \beta_{l}^{*} \lambda=\lambda-e_{j}$ with $j=\max \left\{k \mid \lambda_{k}=l+1\right\}\left(\right.$ so $\left.l=\lambda_{j}-1\right)$.

The $n$-particle hamiltonian has Bethe Ansatz eigenfunctions given by the following plane wave expansion

$$
\phi_{\xi}(\lambda):=\sum_{\substack{\sigma \in S_{n} \\ \epsilon \in\{ \pm 1\}^{n}}} C\left(\epsilon \xi_{\sigma}\right) e^{i\left\langle\lambda, \epsilon \xi_{\sigma}\right\rangle}
$$

with expansion coefficients of the form

$$
\begin{aligned}
C(\xi) & :=\prod_{1 \leq j \leq n} \frac{1-a e^{-i \xi_{j}}+c e^{-2 i \xi_{j}}}{1-e^{-2 i \xi_{j}}} \\
& \times \prod_{1 \leq j<k \leq n}\left(\frac{1-q e^{-i\left(\xi_{j}-\xi_{k}\right)}}{1-e^{-i\left(\xi_{j}-\xi_{k}\right)}}\right)\left(\frac{1-q e^{-i\left(\xi_{j}+\xi_{k}\right)}}{1-e^{-i\left(\xi_{j}+\xi_{k}\right)}}\right) .
\end{aligned}
$$

Here $\langle\cdot, \cdot\rangle$ denotes the standard inner product on $\mathbb{R}^{n}, \epsilon \xi_{\sigma}:=\left(\epsilon_{1} \xi_{\sigma_{1}}, \epsilon_{2} \xi_{\sigma_{2}}, \ldots, \epsilon_{n} \xi_{\sigma_{n}}\right)$, and the summation is meant over all permutations $\sigma$ in the symmetric group $S_{n}$ 
and all sign configurations $\epsilon=\left(\epsilon_{1}, \ldots, \epsilon_{n}\right) \in\{1,-1\}^{n}$. Viewed as a function of the spectral parameter $\xi=\left(\xi_{1}, \ldots, \xi_{n}\right)$ in the fundamental alcove

$$
A:=\left\{\left(\xi_{1}, \xi_{2}, \ldots, \xi_{n}\right) \in \mathbb{R}^{n} \mid \pi>\xi_{1}>\xi_{2}>\cdots>\xi_{n}>0\right\},
$$

the expression $\phi_{\xi}(\lambda), \lambda \in \Lambda_{n}$ amounts to Macdonald's three-parameter Hall-Littlewood polynomial with hyperoctahedral symmetry associated with the root system $B C_{n}$ [M, §10].

Proposition 3.2 (Bethe Ansatz eigenfunctions). The n-particle Bethe Ansatz wave function $\phi_{\xi}, \xi \in A$ solves the eigenvalue equation

$$
H_{q} \phi_{\xi}=E_{n}(\xi) \phi_{\xi}, \quad E_{n}(\xi):=2 \sum_{j=1}^{n} \cos \left(\xi_{j}\right) .
$$

Proof. It follows from Proposition 3.1 that the stated eigenvalue equation boils down to the following identity

$$
\begin{aligned}
a\left[m_{0}(\lambda)\right] \phi_{\xi}(\lambda) & +\sum_{\substack{1 \leq j \leq n \\
\lambda+e_{j} \in \Lambda_{n}}}\left(1-c \delta_{\lambda_{j}} q^{m_{0}(\lambda)-1}\right)\left[m_{\lambda_{j}}(\lambda)\right] \phi_{\xi}\left(\lambda+e_{j}\right) \\
& +\sum_{\substack{1 \leq j \leq n \\
\lambda-e_{j} \in \Lambda_{n}}}\left[m_{\lambda_{j}}(\lambda)\right] \phi_{\xi}\left(\lambda-e_{j}\right)=2 \phi_{\xi}(\lambda) \sum_{j=1}^{n} \cos \left(\xi_{j}\right),
\end{aligned}
$$

which is in turn equivalent to the Pieri formula for the hyperoctahedral HallLittlewood function in Eq. A.3 of Appendix A.

\section{Diagonalization}

From now on it will be assumed unless stated otherwise that $0<|q|<1$ and that the boundary parameters $a$ and $c$ are chosen such that the roots $r_{1}, r_{2}$ of the quadratic polynomial $r^{2}-a r+c$ belong to the interval $(-1,1)$ :

$$
a=r_{1}+r_{2} \text { and } c=r_{1} r_{2} \text { with } r_{1}, r_{2} \in(-1,1) .
$$

Let $L^{2}(A, \Delta \mathrm{d} \xi)$ be the Hilbert space of functions $\hat{f}: A \rightarrow \mathbb{C}$ characterized by the inner product

$$
\langle\hat{f}, \hat{g}\rangle_{\Delta}=\frac{1}{(2 \pi)^{n}} \int_{A} \hat{f}(\xi) \overline{\hat{g}(\xi)} \Delta(\xi) \mathrm{d} \xi, \quad \text { where } \quad \Delta(\xi):=\frac{1}{|C(\xi)|^{2}}
$$

with $C(\xi)$ given by Eq. (3.1b). It is well-known that for the parameter regime in question Macdonald's hyperoctahedral Hall-Littlewood functions form an orthogonal basis of $L^{2}(A, \Delta \mathrm{d} \xi)[\mathrm{M}, \S 10]$ :

$$
\langle\phi(\lambda), \phi(\mu)\rangle_{\Delta}= \begin{cases}\mathcal{N}(\lambda) & \text { if } \lambda=\mu, \\ 0 & \text { otherwise }\end{cases}
$$

where

$$
\mathcal{N}(\lambda):=(c ; q)_{m_{0}(\lambda)} \prod_{\ell \in \mathbb{N}}\left[m_{\ell}(\lambda)\right] !
$$

with $(c ; q)_{m}:=(1-c)(1-c q) \cdots\left(1-c q^{m-1}\right)$ (and the convention that $\left.(c ; q)_{0}:=1\right)$ and $[m] !:=(q ; q)_{m} /(q ; q)_{1}^{m}=[m][m-1] \cdots[2][1]$. By combining the orthogonality 
in Eqs. 4.3a , 4.3b with Proposition 3.2 the spectral decomposition of $\mathrm{H}_{q}$ in the $n$-particle Hilbert space $\ell^{2}\left(\Lambda_{n}, \mathcal{N}^{-1}\right) \subset \mathcal{F}\left(\Lambda_{n}\right)$ characterized by the inner product

$$
\langle f, g\rangle_{n}:=\sum_{\lambda \in \Lambda_{n}} f(\lambda) \overline{g(\lambda)} \mathcal{N}^{-1}(\lambda)
$$

becomes immediate.

Theorem 4.1 (Diagonalization). For $0<|q|<1$ and values of the boundary parameters $a$ and $c$ in the orthogonality domain (4.1), the q-boson Hamiltonian $H_{q}$ (2.4) restricts to a bounded self-adjoint operator in $\ell^{2}\left(\Lambda_{n}, \mathcal{N}^{-1}\right)$ with purely absolutely continuous spectrum. More specifically, its spectral decomposition reads explicitly

$$
H_{q}=\boldsymbol{F}_{\boldsymbol{q}}{ }^{-1} \circ \hat{E} \circ \boldsymbol{F}_{\boldsymbol{q}}
$$

where $\boldsymbol{F}_{\boldsymbol{q}}: \ell^{2}\left(\Lambda_{n}, \mathcal{N}^{-1}\right) \rightarrow L^{2}(A, \Delta d \xi)$ denotes the unitary Fourier transform associated with the hyperoctahedral Macdonald-Hall-Littlewood basis:

$$
\left(\boldsymbol{F}_{\boldsymbol{q}} f\right)(\xi):=\left\langle f, \phi_{\xi}\right\rangle_{n}=\sum_{\lambda \in \Lambda_{n}} f(\lambda) \overline{\phi_{\xi}(\lambda)} \mathcal{N}^{-1}(\lambda)
$$

$\left(f \in \ell^{2}\left(\Lambda_{n}, \mathcal{N}^{-1}\right)\right)$ with the inversion formula given by

$$
\left(\boldsymbol{F}_{\boldsymbol{q}}{ }^{-1} \hat{f}\right)(\lambda)=\langle\hat{f}, \overline{\phi(\lambda)}\rangle_{\Delta}=\frac{1}{(2 \pi)^{n}} \int_{A} \hat{f}(\xi) \phi_{\xi}(\lambda) \Delta(\xi) d \xi
$$

$\left(\hat{f} \in L^{2}(A, \Delta d \xi)\right)$, and $(\hat{E} \hat{f})(\xi):=E_{n}(\xi) \hat{f}(\xi)$ stands for the bounded real multiplication operator in $L^{2}(A, \Delta d \xi)$ associated with the $n$-particle eigenvalue $E_{n}(\xi)$ (3.3).

In the Fock space $\mathcal{H}:=\bigoplus_{n \geq 0} \ell^{2}\left(\Lambda_{n}, \mathcal{N}^{-1}\right)$, built of all linear combinations $\sum_{n>0} c_{n} f_{n}$ with $c_{n} \in \mathbb{C}$ and $f_{n} \in \ell^{2}\left(\Lambda_{n}, \mathcal{N}^{-1}\right)$ such that $\sum_{n>0}\left|c_{n}\right|^{2}\left\langle f_{n}, f_{n}\right\rangle_{n}<$ $\infty$, the $q$-boson hamiltonian $\mathrm{H}_{q}$ (2.4) constitutes an unbounded operator that is essentially self-adjoint on the dense domain $\mathcal{D}:=\mathcal{F} \cap \mathcal{H}$ (because for $z \in \mathbb{C} \backslash \mathbb{R}$ the range $\left(\mathrm{H}_{q}-z\right) \mathcal{D}$ is dense in $\mathcal{H}$ and $\left.\lim _{n \rightarrow \infty} \sup _{\xi \in A}\left|E_{n}(\xi)\right|=\infty\right)$. The representation of the deformed $q$-boson field algebra in Section 2 on the other hand gives rise to a bounded representation on $\mathcal{H}$ :

$$
\begin{aligned}
\left\langle\beta_{l} f, \beta_{l} f\right\rangle_{n-1} & \leq \frac{1+|c| \delta_{l}}{1-q}\langle f, f\rangle_{n} \\
\left\langle\beta_{l}^{*} f, \beta_{l}^{*} f\right\rangle_{n+1} & \leq \frac{1+|c| \delta_{l}}{1-q}\langle f, f\rangle_{n}, \\
\left\langle q^{N_{l}} f, q^{N_{l}} f\right\rangle_{n} & \leq\langle f, f\rangle_{n}
\end{aligned}
$$

preserving the $*$-structure:

$$
\left\langle\beta_{l}^{*} f, g\right\rangle_{n+1}=\left\langle f, \beta_{l} g\right\rangle_{n} \quad \text { and } \quad\left\langle q^{N_{l}} f, g\right\rangle_{n}=\left\langle f, q^{N_{l}} g\right\rangle_{n} .
$$

Remark 4.2. Upon rescaling the lattice $\Lambda_{n}$ (2.2) and performing an appropriate continuum limit [D2, Sec. 5], Macdonald's hyperoctahedral Hall-Littlewood functions tend to the eigenfunctions of the quantum nonlinear Schrödinger equation on the half-line with a boundary interaction [G1, GLM, HL, CC, TW]. In particular, it follows from [D2, Sec. 5.3] that for $a=0$ (which corresponds to a reduction from type $B C$ to type $C$ root systems) a renormalized version of the $q$-boson hamiltonian 
$\mathrm{H}_{q}$ (2.4) then converges in the $n$-particle subspace in the strong resolvent sense to a hamiltonian that can be written formally as:

$$
-\sum_{j=1}^{n} \frac{\partial^{2}}{\partial x_{j}^{2}}+g \sum_{1 \leq j<k \leq n}\left(\delta\left(x_{j}-x_{k}\right)+\delta\left(x_{j}+x_{k}\right)\right)+g_{0} \sum_{1 \leq j \leq n} \delta\left(x_{j}\right)
$$

with $g, g_{0}>0$ (where $\delta(\cdot)$ stands for the 'delta potential').

\section{FACTORIZED SCATTERING}

The similarity transformation

$$
H:=\mathcal{N}^{-1 / 2} \mathrm{H}_{q} \mathcal{N}^{1 / 2}
$$

turns the $n$-particle $q$-boson hamiltonian in Proposition 3.1 into a self-adjoint operator in $\ell^{2}\left(\Lambda_{n}\right)$ diagonalized by the normalized wave function

$$
\begin{aligned}
\Psi_{\xi}(\lambda) & :=e^{\frac{\pi i}{2} n^{2}}|C(\xi)|^{-1} \mathcal{N}(\lambda)^{-1 / 2} \phi_{\xi}(\lambda) \\
& =\mathcal{N}(\lambda)^{-1 / 2} \sum_{\substack{\sigma \in S_{n} \\
\epsilon \in\{ \pm 1\}^{n}}} \operatorname{sign}(\epsilon \sigma) \hat{\mathcal{S}}\left(\epsilon \xi_{\sigma}\right)^{1 / 2} e^{i\left\langle\rho+\lambda, \epsilon \xi_{\sigma}\right\rangle}
\end{aligned}
$$

with $\xi \in A$ (3.2), $\operatorname{sign}(\epsilon \sigma):=\epsilon_{1} \cdots \epsilon_{n} \operatorname{sign}(\sigma), \rho:=(n-1, n-2, \ldots, 2,1,0)$, and

$$
\hat{\mathcal{S}}(\xi):=\prod_{1 \leq j<k \leq n} s\left(\xi_{j}-\xi_{k}\right) s\left(\xi_{j}+\xi_{k}\right) \prod_{1 \leq j \leq n} s_{0}\left(\xi_{j}\right),
$$

where

$$
s(x):=\frac{1-q e^{-i x}}{1-q e^{i x}} \quad \text { with } \quad s(x)^{1 / 2}=\frac{1-q e^{-i x}}{\left|1-q e^{i x}\right|}
$$

and

$$
s_{0}(x):=\frac{1-a e^{-i x}+c e^{-2 i x}}{1-a e^{i x}+c e^{2 i x}} \quad \text { with } \quad s_{0}(x)^{1 / 2}=\frac{1-a e^{-i x}+c e^{-2 i x}}{\left|1-a e^{i x}+c e^{2 i x}\right|} .
$$

Specifically, one has that $H=\boldsymbol{F}^{-1} \circ \hat{E} \circ \boldsymbol{F}$ where $\boldsymbol{F}: \ell^{2}\left(\Lambda_{n}\right) \rightarrow L^{2}(A, \mathrm{~d} \xi)$ denotes the unitary Fourier transformation determined by the kernel $\Psi_{\xi}(\lambda)$ (and $\hat{E}$ is now interpreted as a bounded multiplication operator in $\left.L^{2}(A, \mathrm{~d} \xi)\right)$. For $q, a, c \rightarrow 0$ the $n$-particle $q$-boson hamiltonian $H$ (5.1) simplifies to a hamiltonian modeling impenetrable bosons on $\mathbb{N}$ :

$$
\left(H_{0} f\right)(\lambda)=\sum_{\substack{1 \leq j \leq n \\ \lambda+e_{j} \in \Lambda_{n}}} f\left(\lambda+e_{j}\right)+\sum_{\substack{1 \leq j \leq n \\ \lambda-e_{j} \in \Lambda_{n}}} f\left(\lambda-e_{j}\right)
$$

$\left(f \in \ell^{2}\left(\Lambda_{n}\right)\right.$ ), which is diagonalized by the conventional Fourier transform $\boldsymbol{F}_{\mathbf{0}}$ : $\ell^{2}\left(\Lambda_{n}\right) \rightarrow L^{2}(A, \mathrm{~d} \xi)$ obtained from $\boldsymbol{F}$ by setting $\hat{\mathcal{S}}(\xi) \equiv 1, \mathcal{N}(\lambda) \equiv 1$.

As a very special case of the results in [D3, Sec. 4], it now follows that the waveand scattering operators comparing the $q$-boson dynamics

$$
\left(e^{i t H} f\right)(\lambda)=\frac{1}{(2 \pi)^{n}} \int_{A} e^{i t E_{n}(\xi)} \hat{f}(\xi) \Psi_{\xi}(\lambda) \mathrm{d} \xi \quad \hat{f}=\boldsymbol{F} f
$$

with the corresponding impenetrable boson dynamics generated by $H_{0}$ are governed by a unitary $S$-matrix $\hat{\mathcal{S}}: L^{2}(A, \mathrm{~d} \xi) \rightarrow L^{2}(A, \mathrm{~d} \xi)$ of the form

$$
(\hat{\mathcal{S}} \hat{f})(\xi):=\hat{\mathcal{S}}\left(\epsilon_{\xi} \xi_{\sigma_{\xi}}\right) \hat{f}(\xi) \quad\left(\hat{f} \in C_{0}\left(A_{r}\right)\right.
$$


Here $C_{0}\left(A_{r}\right)$ denotes the dense subspace of $L^{2}(A, \mathrm{~d} \xi)$ consisting of smooth test functions with compact support in the open dense subset $A_{r} \subset A$ for which the components of $\nabla E_{n}(\xi)=\left(-2 \sin \left(\xi_{1}\right), \ldots,-2 \sin \left(\xi_{n}\right)\right)$ do not vanish and are all distinct in absolute value, and the sign-configuration $\epsilon_{\xi}$ and the permutation $\sigma_{\xi}$ are such that the components of $\nabla E_{n}\left(\epsilon_{\xi} \xi_{\sigma_{\xi}}\right)$ are all positive and ordered from large to small. Specifically, by comparing the large-time asymptotics of oscillatory integrals of the form in Eq. (5.3) for the dynamics generated by $H$ and $H_{0}$ one concludes that [D3, Thm. 4.2 and Cor. 4.3]:

Theorem 5.1 (Wave and scattering operators). The operator limits

$$
\Omega^{ \pm}:=s-\lim _{t \rightarrow \pm \infty} e^{i t H} e^{-i t H_{0}}
$$

converge in the strong $\ell^{2}\left(\Lambda_{n}\right)$-norm topology and the corresponding wave operators $\Omega_{r}^{ \pm}$are given by unitary operators in $\ell^{2}\left(\Lambda_{n}\right)$ of the form

$$
\Omega_{r}^{ \pm}=\boldsymbol{F}^{-1} \circ \hat{\mathcal{S}}^{\mp 1 / 2} \circ \boldsymbol{F}_{\mathbf{0}} .
$$

Hence, the scattering operator comparing the dynamics of $H$ and $H_{0}$ is given by the unitary operator

$$
\mathcal{S}:=\left(\Omega_{r}^{+}\right)^{-1} \Omega_{r}^{-}=\boldsymbol{F}_{\mathbf{0}}^{-1} \circ \hat{\mathcal{S}} \circ \boldsymbol{F}_{\mathbf{0}} .
$$

\section{ApPendix A. Pieri formula for Macdonald's hyperoctahedral HALL-LITTLEWOOD FUNCTION}

Let $x:=\left(x_{1}, \ldots, x_{n}\right)=\left(e^{i \xi_{1}}, \ldots, e^{i \xi_{n}}\right)$ and $\tau:=\left(\tau_{1}, \ldots, \tau_{n}\right)$, where $\tau_{j}=r q^{n-j}$ $(j=1, \ldots, n)$ with $r=\frac{a}{2}+\sqrt{\left(\frac{a}{2}\right)^{2}-c}$ (cf. Eq. (4.1)). Upon setting

$$
P_{\lambda}(x):=\frac{\tau_{1}^{\lambda_{1}} \cdots \tau_{n}^{\lambda_{n}}}{\mathcal{N}(0)} \phi_{\xi}(\lambda) \quad\left(\lambda \in \Lambda_{n}\right),
$$

where $\mathcal{N}(0)$ is given by Eq. (4.3b) with $\lambda=0$, the hyperoctahedral Hall-Littlewood function is renormalized to have unital principal specialization values: $P_{\lambda}(\tau)=1$ $\left(\forall \lambda \in \Lambda_{n}\right)[\mathrm{M}, \S 10]$. With this normalization, the following Pieri formula holds:

$$
\begin{aligned}
P_{\lambda}(x) & \sum_{j=1}^{n}\left(x_{j}+x_{j}^{-1}-\tau_{j}-\tau_{j}^{-1}\right)= \\
& \sum_{\substack{1 \leq j \leq n \\
\lambda+e_{j} \in \Lambda_{n}}} V_{j}^{+}(\lambda)\left(P_{\lambda+e_{j}}(x)-P_{\lambda}(x)\right)+\sum_{\substack{1 \leq j \leq n \\
\lambda-e_{j} \in \Lambda_{n}}} V_{j}^{-}(\lambda)\left(P_{\lambda-e_{j}}(x)-P_{\lambda}(x)\right),
\end{aligned}
$$

where

$$
\begin{aligned}
& V_{j}^{+}(\lambda)=\tau_{j}^{-1}\left(\frac{1-c^{2} \delta_{\lambda_{j}} q^{2(n-j)}}{1+c \delta_{\lambda_{j}} q^{2(n-j)}}\right) \prod_{\substack{j<k \leq n \\
\lambda_{k}=\lambda_{j}}}\left(\frac{1-q^{1+k-j}}{1-q^{k-j}}\right)\left(\frac{1+c \delta_{\lambda_{j}} q^{1+2 n-k-j}}{1+c \delta_{\lambda_{j}} q^{2 n-k-j}}\right), \\
& V_{j}^{-}(\lambda)=\tau_{j} \prod_{\substack{1 \leq k<j \\
\lambda_{k}=\lambda_{j}}}\left(\frac{1-q^{1+j-k}}{1-q^{j-k}}\right) .
\end{aligned}
$$

The formula in question is readily obtained through degeneration from an analogous Pieri formula for a $B C_{n}$-type Macdonald function that arises as a special case of the Pieri formulas in [D1, Sec. 6.1]. Specifically, by substituting $t_{2}=q^{1 / 2}$, 
$t_{3}=-q^{1 / 2}$ (which amounts to a reduction from the Macdonald-Koornwinder function to the $B C_{n}$-type Macdonald function) in the Pieri formula of D1, Eqs. (6.4), (6.5)] with coefficients taken from [D1, Eqs. (6.12), (6.13)], the relation in Eq. (A.2) is retrieved for $q \rightarrow 0$ (which corresponds to a transition from Macdonald type functions to Hall-Littlewood type functions). Notice in this connection that the parameters $q, a, c$ (and $r$ ) of the present paper are related to the parameters $t$, $t_{0}, t_{1}$ of Ref. [D1] via $q=t, a=t_{0}+t_{1}, c=t_{0} t_{1}$ (and $r=t_{0}$ ).

Since

$$
V_{j}^{+}(\lambda)=\tau_{j}^{-1}\left(1-c \delta_{\lambda_{j}} q^{m_{0}(\lambda)-1}\right)\left[m_{\lambda_{j}}(\lambda)\right], \quad V_{j}^{-}(\lambda)=\tau_{j}\left[m_{\lambda_{j}}(\lambda)\right],
$$

and

$$
\sum_{j=1}^{n}\left(\tau_{j}+\tau_{j}^{-1}\right)-\sum_{\substack{1 \leq j \leq n \\ \lambda-e_{j} \in \Lambda_{n}}} \tau_{j}\left[m_{\lambda_{j}}(\lambda)\right]-\sum_{\substack{1 \leq j \leq n \\ \lambda+e_{j} \in \Lambda_{n}}} \tau_{j}^{-1}\left[m_{\lambda_{j}}(\lambda)\right]=r\left[m_{0}(\lambda)\right],
$$

the Pieri formula (A.2) can be condensed into the more compact form

$$
\begin{aligned}
P_{\lambda}(x) \sum_{j=1}^{n}\left(x_{j}+x_{j}^{-1}\right) & =a\left[m_{0}(\lambda)\right]+\sum_{\substack{1 \leq j \leq n \\
\lambda-e_{j} \in \Lambda_{n}}} \tau_{j}\left[m_{\lambda_{j}}(\lambda)\right] P_{\lambda-e_{j}}(x) \\
& +\sum_{\substack{1 \leq j \leq n \\
\lambda+e_{j} \in \Lambda_{n}}} \tau_{j}^{-1}\left(1-c \delta_{\lambda_{j}} q^{m_{0}(\lambda)-1}\right)\left[m_{\lambda_{j}}(\lambda)\right] P_{\lambda+e_{j}}(x) .
\end{aligned}
$$

\section{REFERENCES}

BIK. N.M. Bogoliubov, A.G. Izergin, and A.N. Kitanine, Correlation functions for a strongly correlated boson system, Nuclear Phys. B 516 (1998), 501-528.

CC. V. Caudrelier and N. Crampé, Exact results for the one-dimensional many-body problem with contact interaction: including a tunable impurity, Rev. Math. Phys. 19 (2007), 349-370.

D1. J.F. van Diejen, Properties of some families of hypergeometric orthogonal polynomials in several variables, Trans. Amer. Math. Soc. 351 (1999), 233-270.

D2. _ On the Plancherel formula for the (discrete) Laplacian in a Weyl chamber with repulsive boundary conditions at the walls, Ann. Henri Poincaré 5 (2004), 135-168.

D3. Scattering theory of discrete (pseudo) Laplacians on a Weyl chamber, Amer. J. Math. 127 (2005), 421-458.

DE. J.F. van Diejen and E. Emsiz, Diagonalization of the infinite $q$-boson system, preprint 2013.

GLM. M. Gattobigio, A. Liguori, and M. Mintchev, The nonlinear Schrödinger equation on the half line, J. Math. Phys. 40 (1999), 2949-2970.

G1. M. Gaudin, Boundary energy of a Bose gas in one dimension. Phys. Rev. A 4 (1971), 386-394.

G2. La fonction d'onde de Bethe, Masson, Paris, 1983.

G. E. Gutkin, Quantum nonlinear Schrödinger equation: two solutions, Phys. Rep. 167 (1988), 1-131.

HL. M. Hallnäs and E. Langmann, Exact solutions of two complementary one-dimensional quantum many-body systems on the half-line, J. Math. Phys. 46 (2005), no. 5, 052101.

KS. A. Klimyk and K. Schmüdgen, Quantum Groups and their Representations, SpringerVerlag, Berlin, 1997.

KBI. V.E. Korepin, N.M. Bogoliubov, and A.G. Izergin, Quantum Inverse Scattering Method and Correlation Functions, Cambridge University Press, Cambridge, 1993.

K. C. Korff, Cylindric versions of specialised Macdonald functions and a deformed Verlinde algebra, Comm. Math. Phys. 318 (2013), 173-246. 
M. I.G. Macdonald, Orthogonal polynomials associated with root systems, Sém. Lothar. Combin. 45 (2000/01), Art. B45a, 40 pp.

Mt. D.C. Mattis, The Many-Body Problem: An Encyclopedia of Exactly Solved Models in One Dimension, World Scientific, Singapore, 1994.

R. S.N.M. Ruijsenaars, Factorized weight functions vs. factorized scattering, Comm. Math. Phys. 228 (2002), 467-494.

S. B. Sutherland, Beautiful Models: 70 Years of Exactly Solved Quantum Many-Body Problems, Singapore: World Scientific, 2004.

TW. C.A. Tracy and H. Widom, The Bose gas and asymmetric simple exclusion process on the half-line, J. Stat. Phys. 150 (2013), 1-12.

T. N.V. Tsilevich, The quantum inverse scattering method for the $q$-boson model and symmetric functions, Funct. Anal. Appl. 40 (2006), 207-217.

Facultad de Matemáticas, Pontificia Universidad Católica de Chile, Casilla 306, Correo 22, Santiago, Chile

E-mail address: diejen@mat.puc.cl, eemsiz@mat.puc.cl 